\title{
Determination of Effective Thermal Conductivity of Steel Bar Bundles with Use of Krischer Model
}

\author{
R. WyczóŁkowski, D. Urbaniak*, T. Wylecią and H. Otwinowski \\ Czestochowa University of Technology, 42-201 Częstochowa, Poland \\ Doi: $10.12693 /$ APhysPolA.138.317 \\ *e-mail: urbaniak@imc.pcz.pl
}

\begin{abstract}
The article presents an analysis of the possibilities of using the Krischer model to determine the effective thermal conductivity of steel bar bundles for which the solid-phase is low-carbon steel and the gas-phase is air. The results of experimental research were used for this purpose. The analysis was carried out for the temperature range of $0-800^{\circ} \mathrm{C}$. Two solutions of the Krischer model were considered, full and simplified. In the simplified solution, it was assumed that the value of the weighting parameter $f$ depends only on the diameter of the bars. It was shown that the results of the simplified solution differ from the results of the full solution by about $5 \%$. This result shows, that from the point of view of industrial practice, the simplified solution of the Krischer model can be used to determine the effective thermal conductivity of packed bundles of carbon steel rods.
\end{abstract}

topics: steel bar bundle, porous media, effective thermal conductivity

\section{Introduction}

Bar bundles are one example of a porous charge that is encountered during the heat treatment of steel products [1]. This charge has a heterogeneous, two-phase structure characterized by the staggered arrangement of the bars. For this reason, the value that describes the ability of a bundle to heat transfer is the effective thermal conductivity $k_{\text {ef }}[2,3]$. Knowledge of the value of this parameter is necessary to correctly determine the heating time of heat treated bars. A number of analytical models have been proposed to determine the effective thermal conductivity of two-phase media. The most commonly used in this area are five following structural models: the Series, Parallel, Maxwell-Eucken (two forms) and Effective Medium Theory (EMT) $[4,5]$. These models determine the value of the $k_{\text {ef }}$ coefficient of a given two-phase medium on the basis of thermal conductivities $k_{i}$ and volume fractions $v_{i}$ of its individual phases. For this reason listed modes are referred as "rigid" models. It should be noted that Series and Parallel models define the maximum (upper bound) and minimal (lower bound) values for the effective thermal conductivity of any porous material.

As demonstrated, rigid models are not suitable for determining the effective thermal conductivity of a porous charge $[6,7]$. Greater possibilities are offered by the "flexible" models that next to the conductivities $k_{i}$ and volume fractions $v_{i}$ contain an additional parameters. One of the most commonly used models of this type is the Krischer model, that is a weighted harmonic mean of the Series and Parallel models [8]:

$$
k_{\text {ef }}=\left(\frac{1+f}{k_{\mathrm{ef}-\mathrm{P}}}+\frac{f}{k_{\mathrm{ef}-\mathrm{S}}}\right)^{-1},
$$

where $k_{\text {ef }-\mathrm{P}}$ and $k_{\text {ef }-\mathrm{S}}$ are the effective thermal conductivity predicted by the Parallel and Series model, and $f$ is the weighting parameter with values ranging from 0 to 1 . If $f=0$, Krischer model is reduced to the Parallel model, while when $f=1$, Krischer model is reduced to the Series model. Thus, by changing the value of the parameter $f$, it is possible to obtain the value of the coefficient $k_{\text {ef }}$ within the limits set by Serial and Parallel models.

The article presents an analysis of the possibilities of using the Krischer model to determine the effective thermal conductivity of steel bar bundles. Different bar diameters were taken into account. It was also assumed that the thermal conductivity of individual phases of the considered charge in question changes as a function of temperature.

\section{Analysis and modelling}

For the analysis of the problem considered in the article, the results of experimental research of the effective thermal conductivity were used [6]. The measurement results showed that the effective thermal conductivity of the samples rises linearly with temperature. Therefore, the measurement results for individual samples were approximated using the least squares method by means of linear regression equations:

$$
k_{\mathrm{ef}}=B_{1}+B_{2} t .
$$


TABLE I

The values of $k_{s}, k_{g}$ coefficients and $k_{s} / k_{g}$ ratio.

\begin{tabular}{c|c|c|c}
\hline \hline Temp. $\left[{ }^{\circ} \mathrm{C}\right]$ & $k_{s}$ & $k_{g}$ & $k_{s} / k_{g}$ \\
\hline 0 & 51.3 & 0.024 & 2137 \\
200 & 47.7 & 0.039 & 1225 \\
400 & 42.1 & 0.051 & 816 \\
600 & 35.1 & 0.062 & 567 \\
800 & 27.3 & 0.069 & 390
\end{tabular}

Temperature changes of thermal conductivity of solid phase $k_{s}$ and gas phase $k_{g}$ for the considered charge are described by the following equations [9]:

$$
\begin{aligned}
& k_{s}=1.2 \times 10^{-8} t^{3}-3.2 \times 10^{-5} t^{2} \\
& \quad-1.2 \times 10^{-2} t+51.3 \\
& k_{g}=-2.88 \times 10^{-8} t^{2}+8.05 \times 10^{-5} t+0.024
\end{aligned}
$$

Table I summarizes the values of the $k_{s}$ and $k_{g}$ coefficients for selected temperatures and the values of the $k_{s} / k_{g}$ ratio. As can be seen, the thermal conductivity values of individual bundle phases are very different from each other, showing a different tendency of change in the function of temperature. In the lower temperature bound, the thermal conductivity of the solid phase is over two thousand times that of the gas phase, while in the upper bound, this ratio drops to 390 .

Using (2)-(4), it is possible to calculate the change of weighting parameter $f$ from the Krischer model for the considered temperature range. The appropriate equation is obtained by transforming the relationship (1) into:

$$
f=\left(\frac{k_{\mathrm{ef}-\mathrm{P}}}{k_{\mathrm{ef}}}-1\right)\left(\frac{k_{\mathrm{ef}-\mathrm{P}}}{k_{\mathrm{ef}-\mathrm{S}}}-1\right)^{-1},
$$

The results of calculations of the parameter $f$ obtained for the analyzed bar bundles are presented in Fig. 1. The value of this parameter decreases as the diameter of the bars increases. While, the increase in temperature initially increases the $f$ value, which takes place to a temperature of about $400^{\circ} \mathrm{C}$. For higher temperatures, a decrease in $f$ value is observed. The curves illustrating the temperature changes of the parameter $f$ can be described by the following polynomial:

$$
f=C_{1} t^{2}+C_{2} t+C_{3} .
$$

The minimum, mean and maximum values of the parameter $f$ obtained for individual bar diameters are presented in Table II.

The presented results show that the Krischer model allows very precisely adjusting the $k_{\text {ef }}$ coefficient of steel bar bundles. However, achieving such compliance requires careful adjustment of the value of the parameter $f$. This adjustment must take into account both the influence of temperature and the diameter of the bars, which is unfortunately not easy. The solution obtained with this adjustment was called the full solution.

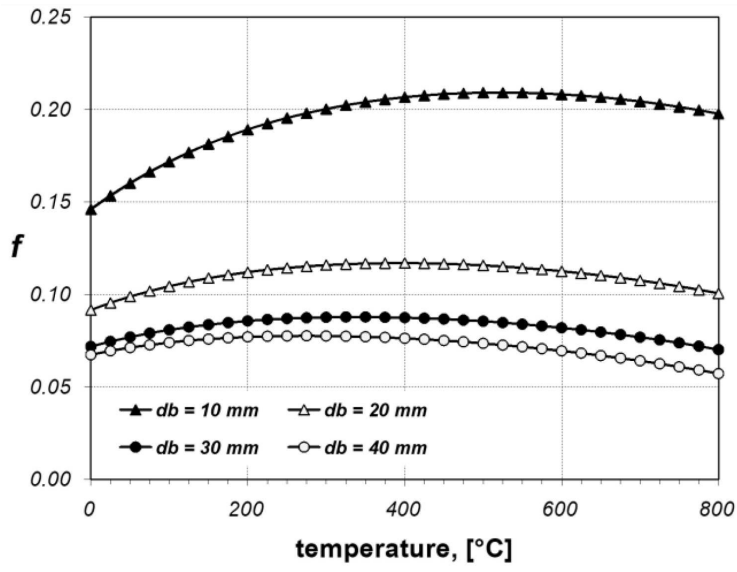

Fig. 1. Values of the parameter $f$ depending on the temperature and bar diameter $d_{b}$.

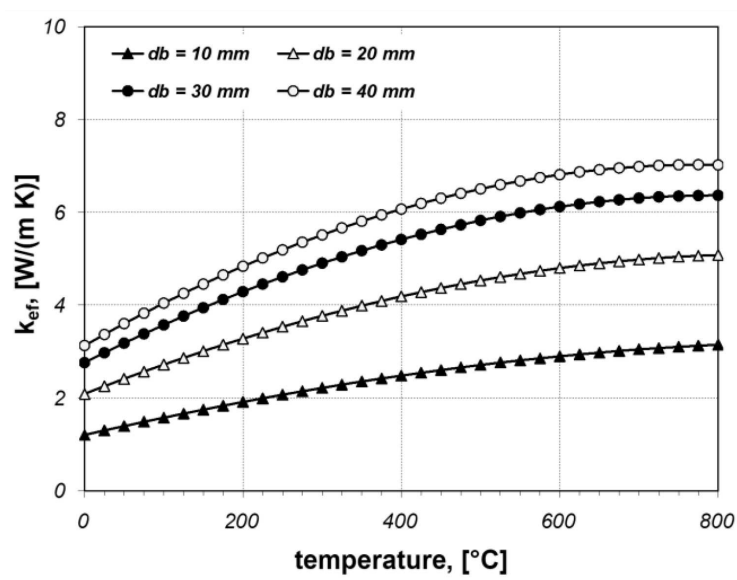

Fig. 2. Effective thermal conductivity of steel bar bundles calculated for constant values of parameter $f$.

TABLE II

Minimum, mean and maximum values of the parameter $f$ depending on the bar diameter.

\begin{tabular}{c|c|c|c}
\hline \hline$d_{b}[\mathrm{~mm}]$ & Min. & Mean & Max. \\
\hline 10 & 0.1461 & 0.1948 & 0.2091 \\
20 & 0.0916 & 0.1099 & 0.1169 \\
30 & 0.0702 & 0.0819 & 0.0877 \\
40 & 0.0572 & 0.0716 & 0.0716
\end{tabular}

Due to the complexity of adjustment the correct value of $f$, it was decided to check the results of the Krischer model assuming that the value of this parameter depends only on the diameter of the bars. For this purpose, calculations were made assuming constant values of $f$ for individual bar diameters for which the mean values from Table II were used. This solution was called a simplified one.

The effective thermal conductivity of bar bundles calculated in a simplified manner is shown in Fig. 2. In this case, the waveforms change of the $k_{\mathrm{ef}}$ parameter as a function of temperature are not straight, as in Fig. 2. Effective thermal conductivity 


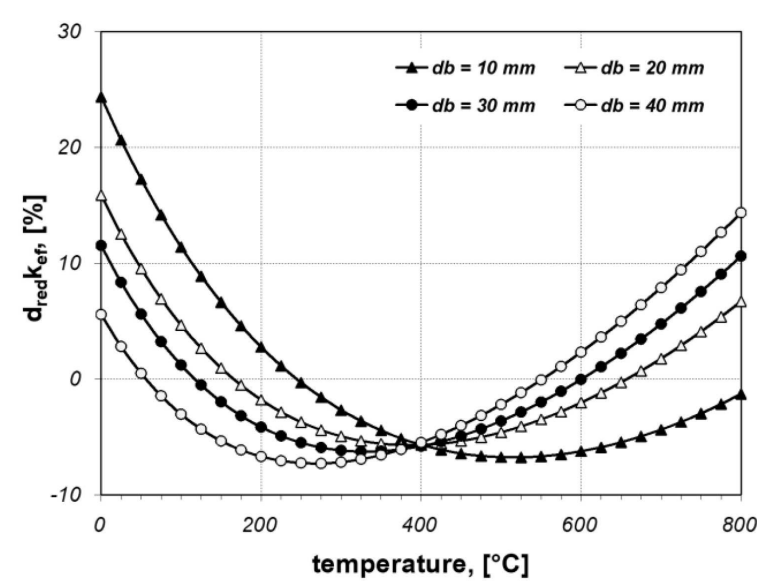

Fig. 3. Calculation results of the reduced difference $d_{\text {red }} k_{\text {ef }}$.

TABLE III

Mean absolute values of the $d_{\text {red }} k_{e f}$ parameter.

\begin{tabular}{c|c|c|c|c}
\hline \hline$d_{b}[\mathrm{~mm}]$ & 10 & 20 & 30 & 40 \\
\hline$d_{\text {red }} k_{\text {ef }}[\%]$ & 6.61 & 4.54 & 4.72 & 5.42
\end{tabular}

increases as a function of temperature, however, these changes are less and less intense with increasing temperature.

To better visualize the divergence of results obtained for a full and simplified solution, the reduced difference was calculated:

$$
d_{\text {red }} k_{\text {ef }}=\frac{k_{\text {ef }-\mathrm{f}}-k_{\text {ef }-\mathrm{s}}}{k_{\text {ef }-\mathrm{f}}} \times 100 \%,
$$

The calculation results of the $d_{\text {red }} k_{\text {ef }}$ parameter are presented in Fig. 3. For the most part of the temperature range, this parameter value does not exceed $\pm 10 \%$. Higher values occur only for 10 and $20 \mathrm{~mm}$ bar. For $10 \mathrm{~mm}$ bars, the maximum value of $d_{\text {red }} k_{\text {ef }}$ occurs at $0{ }^{\circ} \mathrm{C}$, and it is less than $25 \%$, but at $120^{\circ} \mathrm{C}$ it falls below $10 \%$. In turn, for $20 \mathrm{~mm}$ bars the maximum value was $16 \%$ (for $t=0{ }^{\circ} \mathrm{C}$ ), however, after exceeding $50^{\circ} \mathrm{C}$ its value drops below $10 \%$. Exceeding $10 \%$ is still present for $40 \mathrm{~mm}$ bars at temperature above $730{ }^{\circ} \mathrm{C}$ with a maximum value of $14 \%$. Mean absolute values of the $d_{\text {red }} k_{\text {ef }}$ parameter obtained for individual bar diameters are summarized in Table III. As can be seen, the values are much less than $10 \%$, and for bars with a diameter of $20 \mathrm{~mm}$ and $30 \mathrm{~mm}$ even below $5 \%$.

The results summarized in Table III show that for the industrial purposes, the Krischer model with a simplified solution can be used to determine the effective thermal conductivity of low carbon steel bar bundles.

In order to make this solution more general, it was decided to determine a function approximating the value of the parameter $f$ for any diameter of bars in the range from 10 to $40 \mathrm{~mm}$. For this purpose, the mean values of the $f$ parameter were used, presented in Table II. The equation that best matches the calculation results to these values $\left(R^{2}=0.992\right)$ has the form of a power function:

$$
f=1.034 d_{b}^{-0.736},
$$

\section{Conclusions}

The Krischer model is a relatively simple model of effective thermal conductivity, which provides flexibility in calculation by using the weighting parameter $f$. The analyzes presented in the article indicate that the model can be used to determine the effective thermal conductivity of steel bar bundles. A full solution in which the weighting parameter changes as a function of the charge temperature and the diameter of the bars is difficult to obtain. A simplified solution is much more convenient, in which the $f$ changes only as a function of bar diameter. Comparing our results with the experimental studies which are the most reliable source of information on the value of the $k_{\text {ef }}$ coefficient, it was determined that for the considered charge, the changes of the $f$ parameter in the function of $d_{p}$ can be described quite accurately by a power equation. The values of $k_{\mathrm{ef}}$ calculated in this way differ from the value of the full solution by $\approx 5 \%$. From the point of view of industrial practice, such accuracy are completely satisfactory.

\section{References}

[1] C. Kolmasiak, T. Wyleciał, Metalurgija 57, 368 (2018).

[2] M. Kaviany, Principles of heat transfer in porous media, 2nd ed., Springer-Verlag, New York 1995.

[3] W. Van Antwerpen, C.G. du Toit, P.G.Rousseau, Nuc. Eng. Design 240, 1803 (2010).

[4] J.K.Carson, S.J. Lovatt, D.J. Tanner, A.C. Cleland, Int. J. Heat Mass Transf. 48, 2150 (2005).

[5] J.F. Wang, J. K.Carson, J. Willix, M.F. North, D.J. Cleland, Int. J. Refrig. 32, 556 (2009).

[6] R. Wyczolkowski, "Modelling of effective thermal conductivity of the steel porous charge using the thermal resistance concept", Series Monographs, Vol. 67, Czestochowa University of Technology, Czestochowa 2017 (in Polish).

[7] R. Wyczolkowski, A. Benduch, J. Phys. Sci. App. 6, 55 (2016).

[8] J.K. Carson, Int. J. Refrig. 29, 958 (2006).

[9] R. Wyczolkowski, Proc. Eng. 157, 185 (2016). 